\title{
Correction to: Calderas
}

\section{Correction to:}

Chapter 5 in: V. Acocella, Volcano-Tectonic Processes, Advances in Volcanology, https://doi.org/10.1007/978-3-030-65968-4_5

In the original version of the book, the following belated correction have been incorporated in the chapter "Calderas": Fig. 5.13 has been replaced with the correct figure.

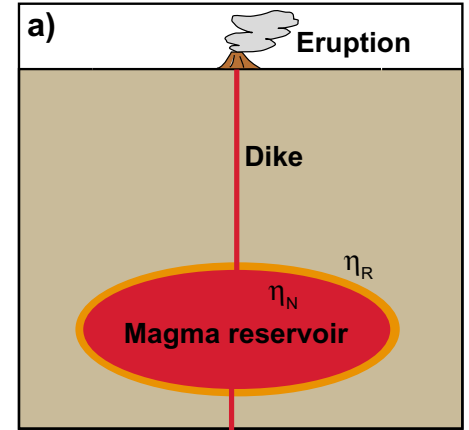

Thinner residual magma

High viscosity contrast $\left(\eta_{R} / \eta_{N}>10^{11}\right)$

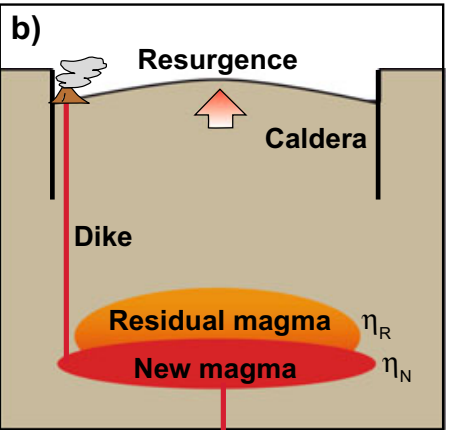

Thicker deep residual magma Relatively low viscosity contrast $\left(\eta_{R} / \eta_{N}<10^{9}\right)$

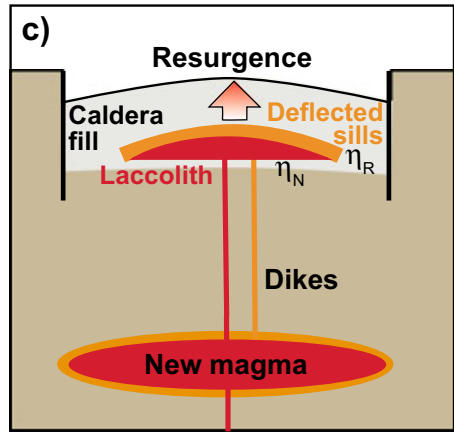

Thicker shallow residual magma Relatively low viscosity contrast $\left(\eta_{R} / \eta_{N}<10^{9}\right)$

Residual magma with viscosity $\eta_{R}$

New magma with viscosity $\eta_{N}$

Fig. 5.13 Conditions for resurgence. a A thinner layer of viscous residual magma (high viscosity contrasts with the new magma) promotes dike propagation and eruption, without resurgence. $\mathbf{b}$ A thicker layer of medium viscosity residual magma (with relatively low viscosity contrast with the new magma) hinders dike propagation, promoting stagnation and resurgence; peripheral eruptions may occur. c Same as (b), but shifted at shallower levels: dikes (orange) may arrest within the altered intracaldera tuff, developing one or more sills (orange) constituting a rheological barrier for successive dikes (red), stagnating in laccoliths and promoting resurgence. Mechanism (c) may be alternative to, or combined with, (b) (modified after Galetto et al. 2017)

The updated version of this chapter can be found at https://doi.org/10.1007/978-3-030-65968-4_5 